\title{
Histological characteristics of hair follicle pattern in Indian bison (Bos gaurus), Black buck (Antelope cervicapra) and Nilgai (Boselaphus tragocamelus)
}

\author{
Y. B. Shambhulingappa ${ }^{1}$, R.V.Prasad ${ }^{1}$, K. V. Jamuna ${ }^{1}$, H. D. Narayanaswamy ${ }^{2}$, M. Narayana Bhat ${ }^{3}$ and V. Ramkrishna $^{1}$
}

1. Department of Veterinary Anatomy and Histology, Veterinary College, Hebbal, Bangalore - 560024, Karnataka, India; 2. Ex-Director, Institute of Wildlife Veterinary Research, KVAFSU, Kudige, Kodagu District, Karnataka, India;

3. Department of Veterinary Pathology, Veterinary College, Hebbal, Bangalore - 560024, Karnataka, India Corresponding author: R. V.Prasad, email: rvprashwin@gmail.com, Mobile: +91-9449855893

Received: 22-01-2014, Revised: 08-03-2014, Accepted: 10-03-2014, Published online: 29-03-2014

doi: 10.14202/vetworld.2014.189-193 How to cite this article: Shambhulingappa YB, Prasad RV, Jamuna KV, Narayanaswamy HD, Bhat MN and Ramkrishna V (2014) Histological characteristics of hair follicle pattern in Indian bison (Bos gaurus), Black buck (Antelope cervicapra) and Nilgai (Boselaphus tragocamelus), Veterinary World 7(3): 189-193.

\begin{abstract}
Background and Aim: Indian bison, Black buck and Nilgai are in danger of extinction because people are hunting them for their meat and horns for sports, trophies etc. These animals are also protected under International Union for Conservation of Nature and Natural Resources (IUCN) and Conservation on International trade in Endangered Species of Wild Fauna and Flora (CITES). Taking note of the vulnerability of these animals to extinction, we characterized the hair follicle pattern of these animals for future reference. This study included a total of 30 skin samples comprised of 8 samples of bison, 10 samples of black buck and 12 samples of nilgai.
\end{abstract}

Materials and Methods: Skin samples were obtained from Sri Jayachamarajendra Zoological Park, Mysore, Karnataka, India and Bannerghatta Biological Park, Bangalore, Karnataka, India and also from confiscated skins from forest and police officials of Karnataka. The skin samples were processed and horizontal section of 6-7 $\mu \mathrm{m}$ thickness was used for the routine histological technique using different special staining techniques. Observation such as hair follicle pattern, sebaceous glands and sweat glands distribution, blood vessels, nerve fibre, collagen and elastic fibres were noted.

Results: In the bison, compound hair follicles were uniformly distributed and were rectangular in shape comprising of a primary hair follicle associated with 3-4 secondary hair follicles in addition to their sebaceous glands. Uniformly distributed coiled tubular sweat glands were also noticed which were lined by simple cuboidal epithelium. In the black buck, the compound hair follicles were densely distributed and were arranged linearly in the dermis of skin. Within the compound follicle, linear arrangements of primary and secondary follicles were observed. Each primary hair follicle was supported by 2 4 secondary hair follicles and the primary hair follicle was always present at the centre when it was present with 2 secondary hair follicles. In nilgai, the compound hair follicles were densely distributed in the dermis of skin. The primary hair follicles were bilaterally surrounded by 2-3 secondary hair follicles. Each primary and secondary hair follicle was associated with sebaceous glands.

Conclusion: The histological pattern of the hair follicle data was established and the hair follicle pattern will be of use in the identification of the wild ruminant skins which are commonly poached by the poachers. It will also be useful to compare the histological characteristics amongst the wild ruminants.

Keywords: biological park, hair follicle, sebaceous glands, sweat glands, wild ruminants.

\section{I ntroduction}

The gaur (Bos gaurus), is also called Indian bison, the largest and tallest species of wild cattle and are in danger of extinction, because people are hunting them for meat, horns for decorative items and internal organs for traditional medicinal purposes [1]. The number of wild gaurs decreased dramatically due to the loss of suitable habitat, hunting or hybridization with domestic cattle [2]. Gaurs were once distributed throughout the forested tracts of India and South Nepal, east to Vietnam and south to Malaya but today they are confined to just over a hundred existing and 27 proposed Protected Areas in India [6]. The species is listed under the IUCN Red List as vulnerable and according to

Copyright: The authors. This article is an open access article licensed under the terms of the Creative Commons Attribution License (http://creativecommons.org/licenses/by/2.0) which permits unrestricted use, distribution and reproduction in any medium, provided the work is properly cited.
Indian Wildlife Protection Act (1972) it is an endangered animal per Schedule-I and also it is included in Appendix I of the Conservation on International trade in Endangered Species of Wild Fauna and Flora (CITES) and categorized as Vulnerable by the International Union for Conservation of Nature and Natural Resources (IUCN) [3, 4]. The black buck (Antelope cervicapra) is one of 26 species of mammals, which have been declared endangered and is the only living species of the genus Antelope [5]. Poaching and habitat destruction have seriously affected the population of the Black Bucks. It is usually hunted for its skin, flesh and also for sports. As its range has decreased sharply during the 20th century, it has been classified as near threatened by IUCN since 2003 [3]. During the $18^{\text {th }}$ century to first half of the 20th centuries, blackbucks were the most hunted wild animal all over India. The nilgai (Boselaphus tragocamelus) 
has become extinct in Bangladesh, it is the only member of genus Boselaphus and the main threat to this species is the loss of habitat due to deforestation and human population growth [5]. Half of the top 20 countries for numbers of threatened species are in Asia. Habitat loss, affecting over 2000 mammal species, is the greatest threat globally [7]. The arrangement of hair follicles in the domestic animals such as cattle, sheep, goat, dog and cat and wild carnivores such as Bengal tiger, leopard, lion and sloth bear have been described and indicated that they have species specific arrangement of hair follicles $[8,9,10]$. Review of literature has not shown any detail on the architecture of hair follicle in wild herbivore animals. Keeping this in view, the present study has been conducted on the original skin of wild herbivores such as Indian gaur, black buck and nilgai.

This study will help in the identification of fake skin from the original herbivore skins which are sold at an exorbitant price by the poachers in the international market.

\section{Materials and Methods}

Ethical approval: Permission obtained from Principal Chief Conservator of Forest (Wildlife) and Chief Wildlife Warden, Karnataka State, Govt. of Karnataka dated 12.09.2012 (No. C1/WL/CR-49/2012-13).

The study of morphological features of hair follicle pattern of the skin of bison, black buck and nilgai were undertaken in the Department of Anatomy and Histology, Veterinary College, Bangalore. The samples were collected from Sri Jayachamarajendra Zoological Gardens, Mysore and Bannerghatta Biological Park, Bangalore, Karnataka. A total of 30 samples of which 8 samples from bison, 10 samples from black buck and 12 samples from nilgai were taken for the present study. These skin specimens were obtained from animals that died of natural causes. During post mortem from the lateral abdomen and leg regions, the skin samples were cut into $2 \times 2 \mathrm{~cm}$ size and fixed in $10 \%$ Neutral buffered formalin for a minimum of 48 hours [11].

Further, they were processed by manual tissue processor and dehydrated in increasing concentration of ethanol and cleared in benzene and embedded in paraffin wax with ceresin $\left(60^{\circ} \mathrm{C}\right.$ melting point). The processed tissues were horizontally sectioned in Microm HM 325 rotary microtome at 6-7 $\mu \mathrm{m}$ thickness. The sections were stained with $H \& E$ Phloxine for morphological studies [12] by deparaffinizing the tissue samples in xylene I \& II and hydrated to distilled water by decreasing grade of alcohol (Absolute I, Absolute II, $95 \%, 70 \%$ and 50\%), then sections were stained with Mayer"s haematoxylin solution for 5 minutes and washed in running water for 15 minutes and rinsed in distilled water. Sections were stained with eosine-phloxine solution for 1 minute, dehydrated in 95\% alcohol, Absolute I, Absolute II and cleared in xyline I \& II then mounted with D.P.X mountant.
Weigerts resorcine fuchsine stain for elastic fibres was done as follows. The sections were deparaffinized in xyline and hydrated to distilled water. Following that, sections were stained with Weigert's hematoxylin (A\&B solutions were mixed in equal quantities to make working solution) then washed well in water to intensify the stain and treated with resorcin-fuchsin solution for 30 minutes and rinsed in $95 \%$ alcohol. Tissues were then washed in water and kept in Van Gieson's solution for a minute, dehydrated in 95\% alcohol, absolute alcohol and cleared in xyline (two changes) then mounted with D.P.X mountant. Masson's Trichrome stain for collagen fibres was carried out by deparaffinizing in xyline and hydrated to distilled water and mordant in bouin's solution overnight at room temperature and then washed in running water until yellow colour disappears. This was followed by rinsing the tissues in distilled water, treated with biebrich scarlet- acid solution (biebrich scarlet, aqueous 1\%-90 ml; acid fuchsin, aqueous $1 \%$ $10 \mathrm{ml}$ and glacial acetic acid, $1 \mathrm{ml}$ ) for 2 minutes and rinsed in distilled water, phosphormolybdic-phosphotungstic acid (phosphormolybdic acid $5 \mathrm{gm}$ and phosphotungstic acid $5 \mathrm{gm}$ were dissolved in $200 \mathrm{ml}$ of distilled water) solution for 15 minutes then sections were directly treated with aniline blue solution for 3 minutes and rinsed in distilled water. Tissues were transferred to glacial acetic acid solution for 3-5 minutes and dehydrated in $95 \%$ alcohol, absolute alcohol and cleared in xyline (two changes) then mounted with D.P.X mountant [13].

Gomori's staining method for reticulum was done by deparaffinizing in xyline and hydrated to distilled water, oxidised in potassium permanganate solution for 1 minute, washed in tap water for 2 minutes, then differentiated with potassium meta bisulfate solution for 1 minute. Then tissue sections were washed in tap water for 2 minutes, sensitized in ferric ammonium sulphate for 1 minute and washed in tap water for 2 minutes along with 2 changes of distilled water. Following that, tissues were impregnated in the silver solution for 1 minute, rinsed in distilled water, reduced in formalin solution for 3 minutes, washed in tap water for 3 minutes, then toned in gold chloride solution for 10 minutes, rinsed in distilled water, after this tissues were reduced in potassium meta bisulfate solution for 1 minute, fixed in sodium thiosulfate solution for a minute, washed in tap water for 2 minutes. Finally tissues were dehydrated in $95 \%$ alcohol, absolute alcohol and cleared in xyline (two changes) then mounted with D.P.X mountant, [13].

Because of various thickness of the skin of wild animals, before routine histological technique, fixed skin samples were washed in running water for overnight and immersed in $4 \%$ aqueous solution of phenol for 3-5 days for softening of skin samples such as black buck and nilgai and 6\% aqueous solution of phenol for thick samples such as bison for 8-10 days. The protocol has been modified as specified in Lendrum's technique [11]. 


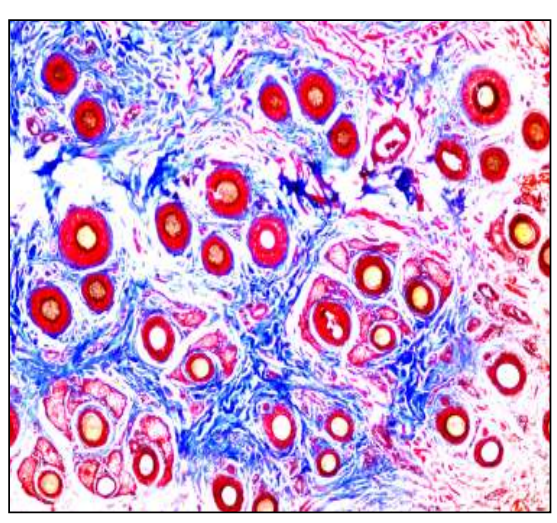

Figure-1: Photomicrograph of horizontal section of Indian bison skin showing uniformly distributed Compound hair follicles. Masson's Trichrome (X40)

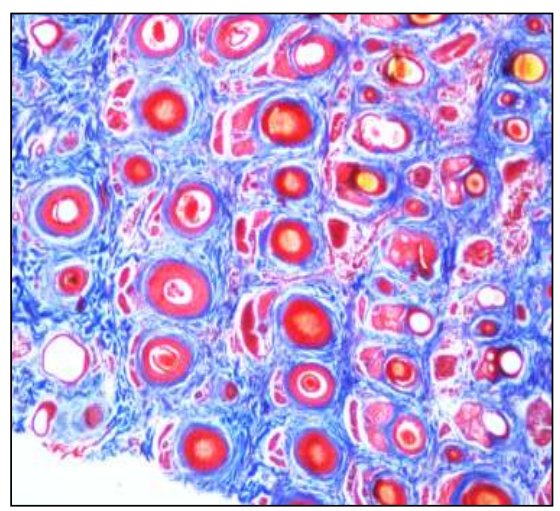

Figure-4: Photomicrograph of horizontal section of black buck skin showing linearly arranged densely distributed compound hair follicles. Masson's Trichrome (X40)

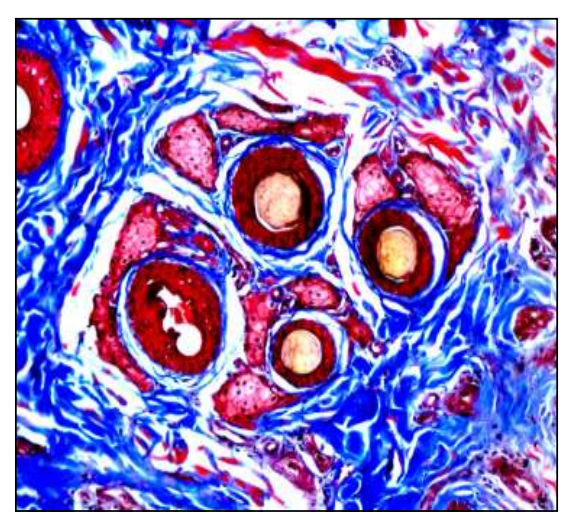

Figure-2: Photomicrograph of horizontal section of Indian bison skin showing rectangular shape compound hair follicle comprising of primary hair follicle associated with 3-4 secondary hair follicles together with their sebaceous glands. Masson's Trichrome (X40)

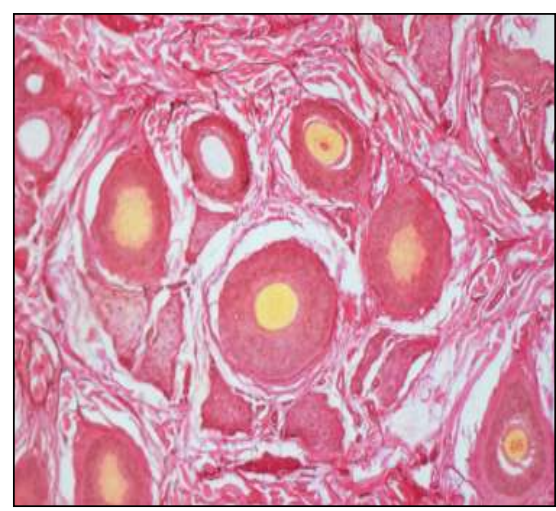

Figure-5: Photomicrograph of horizontal section of black buck skin showing compound hair follicle consists of primary hair follicle with 2-4 secondary hair follicles. Weigert's (X 100)

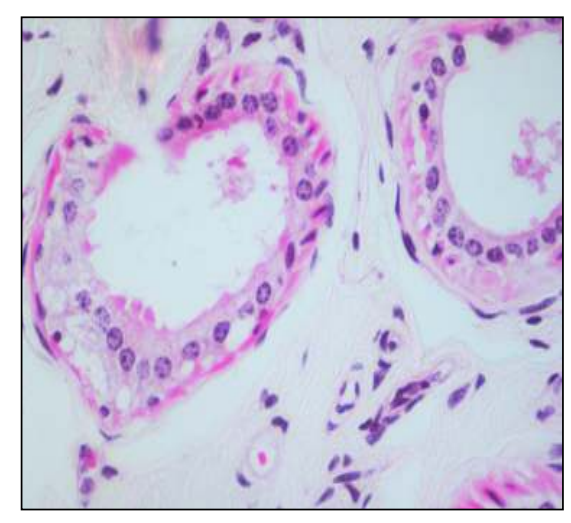

Figure-3: Photomicrograph of horizontal section of Indian bison skin showing coiled tubular sweat glands exhibited secretary blebs on their cell line surface suggesting apocrine mode of secretion. $\mathrm{H}$ and $\mathrm{E}$ ( $\mathrm{X} 400)$

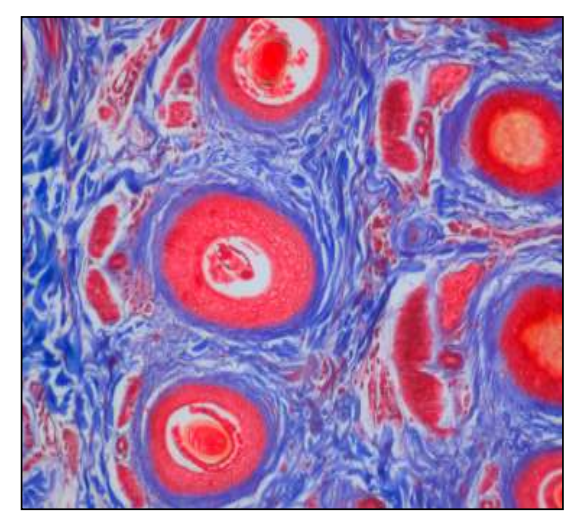

Figure-6: Photomicrograph of horizontal section of black buck skin showing the primary hair follicle present at the centre when it was present with 2 secondary hair follicles with sebaceous glands. Masson's Trichrome (X100)

\section{Results}

Histological structure of the skin of wild ruminants like bison, black buck and nilgai were studied under light microscope. In the bison, compound hair follicles were uniformly distributed (Figure-1) and were rectangular in shape comprising of primary hair follicle associated with 3-4 secondary hair follicles together with their sebaceous glands (Figure2 ). In addition, uniformly distributed coiled tubular sweat glands were also noticed which were lined by simple cuboidal epithelium. The lining cells of sweat glands exhibited secretary blebs on their surface, suggesting apocrine mode of secretion (Figure-3). The collagen fibres were abundant compared to smooth muscle fibre distribution in the dermis. The capillary plexus and fine arterioles and venules comprising the vascular component of the dermis were also noted. Evenly distributed fine elastic fibres were present and reticular fibres were not observed within the connective tissue stroma of dermis.

In black buck, the compound hair follicles were densely distributed and were arranged linearly in the dermis of skin (Figure-4). Within the compound follicle there was linear arrangement of primary and secondary follicles observed. Each primary hair follicle was supported by 2-4 secondary hair follicles (Figure-5) and the primary hair follicle was always present at the centre when it was present with 2 secondary hair follicles (Figure-6). Sebaceous glands were associated with each hair follicle and numerous coiled tubular sweat glands were also present in the dermis of skin (Figure-7). Elastic fibres were uniformly distributed among the collagen fibres and also around the hair follicles. The smooth muscle fibres were sparsely distributed between the collagen fibres and the reticular fibres were absent.

In nilgai, the compound hair follicles were densely distributed in the dermis of skin. The primary hair follicles were bilaterally surrounded by 2-3 secondary hair follicles and each primary and secondary hair follicle was associated with sebaceous glands (Figure-8). The dermal connective tissue comprised of bundles of collagen and smooth muscle fibres separating the hair follicle. The fine strands of elastic fibres and reticular fibres were present in the connective tissue (Figure-9). 


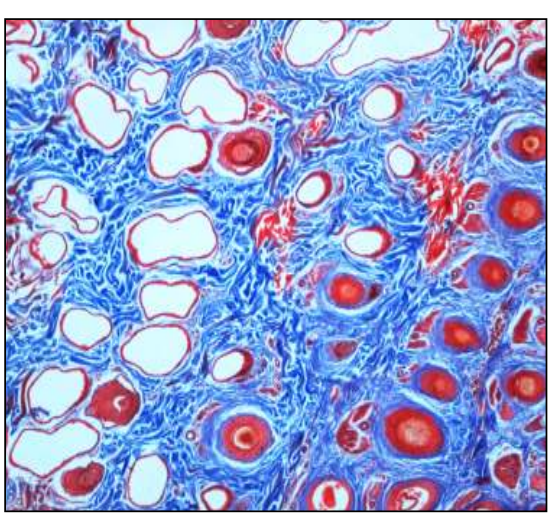

Figure-7: Photomicrograph of horizontal section of black buck skin showing coiled tubular sweat glands in the dermis of skin. Masson's Trichrome (X 40)

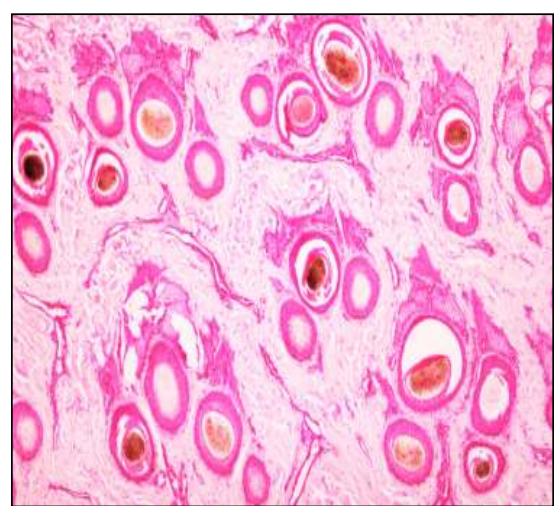

Figure-8: Photomicrograph of horizontal section of nilgai skin showing the primary hair follicles with bilaterally surrounded 2-3 secondary hair follicles and sebaceous glands. $H \& E(X 40)$

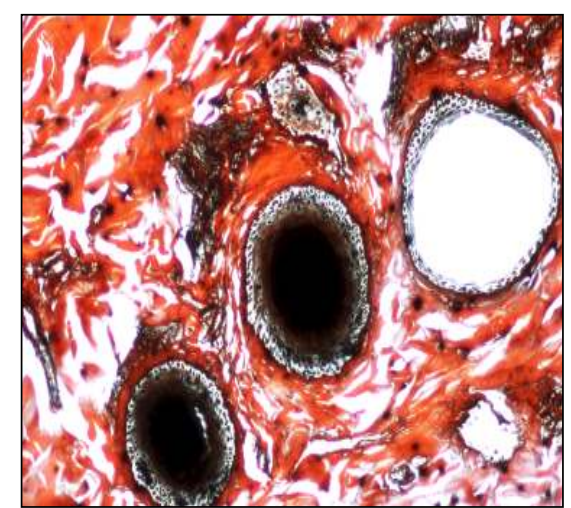

Figure-9: Photomicrograph of horizontal section of nilgai skin showing reticular fibres in the connective tissue. Gomori's (X 100)

\section{Discussion}

The compound hair follicle with a primary hair follicle associated with two to three secondary hair follicles in spotted deer which were arranged in a parallel fashion to each other and the sebaceous glands surrounding the primary hair follicle alone was seen in spotted deer, cattle and goat [9]. However, in the present study the primary hair follicle was associated with 3-4 secondary follicles in bison and bilaterally surrounded 2-3 secondary hair follicles in nilgai which were uniformly distributed in the skin but not arranged in parallel fashion as observed in spotted deer. All the hair follicles were associated with sebaceous glands. In black buck, the compound hair follicles were densely distributed and were arranged linearly as observed in spotted deer and each primary hair follicle was supported by 2-4 secondary hair follicles with sebaceous glands. The primary hair follicle was always present at the centre when it was present with 2 secondary hair follicles. However in Iraqi goats, sebaceous glands were associated with all primary and some secondary follicles [14].

Both simple and compound hair follicle and the dorsal and lateral aspects of the body presented high density of hair follicles with one or two primary hair follicles surrounded by multiple smaller secondary follicles and oriented at a sharp oblique angle in llama skin [15] but there were no oblique orientation of the hair follicle in the wild ruminants in present study. However in the present study there was only one primary hair follicle situated in the compound hair follicle. European cattle having primary hair follicle with no secondary hair follicle has also been reported [16]. Presence of primary hair follicle, secondary hair follicle and sebaceous glands were reported in nonwoolly Indian goats [17] but their distribution pattern was not described.

The coiled tubular sweat glands which were lined by simple cuboidal epithelium with secretory blebs on their surface suggested apocrine mode of secretion in the bison and black buck in the present study. Both bilobed sebaceous glands and sweat glands in the skin of Raini goats were described [18]. The simple tubular sweat glands in the spotted deer, cattle and goat which were located at the junction of dermis and hypodermis with merocrine mode of secretion have been described [9]. However sebaceous glands are associated with secondary hair follicle deep in the dermis of goat skin [19] but both sebaceous and sweat glands associated with hair follicle in red kandhari cows have been reported [20]. However, no sweat glands were observed in nilgai skin in the present study. The dermal connective tissue comprised of bundles of collagen, elastic and smooth muscle fibres in all the wild animals in the present study but reticular fibres were not observed in all the animals except nilgai. In hippopotamus skin, reticular dermis showed very thick bundles of coarse collagen fibres arranged in a more regular pattern and subdermal glands were also observed [21].

\section{Conclusion}

The histological study of Indian bison, black buck and nilgai skins were established. The above observations and description of hair follicle pattern will be useful in the identification of the wild ruminants skin which are poached by the poachers. Observation made by this study will help wildlife students, pathologists and researchers for academic purposes and solving the vetero-legal cases.

\section{Authors' contributions}

YBS and RVP designed the study and initiated the research. MNB and VR carried out collection and preservation of samples from the national park and zoo during the study, KVJ helped in standardi-sation of procedure, YBS and HDN helped in preparation of tissue blocks and photography. RVP and KVJ helped in draft and revision of manuscript, interpretation of results, All authors read and approved the final manuscript.

\section{Acknowledgements}

The authors are thankful to the Principal Chief 
Conservator of Forest (Wildlife Warden) Government of Karnataka, for issuing the permission letter to collect the skin samples for the study. The authors are also thankful to Executive Directors, Veterinarians and supporting staff of Sri Jayachamarajendra Zoological Park Mysore and Bannerghatta National Park Bangalore, Karnataka for helping in the collection of samples during the course of study. The authors acknowledge the financial support and facilities provided for present study from the Karnataka Veterinary Animal and Fisheries Sciences University, Bidar, Karnataka.

\section{Competing interests}

The authors declare that they have no competing interests.

\section{References}

1. Menon and Vivek. (2009) Field Guide to Indian Mammals, Christopher Helm Publishers. ISBN 978-1408112137. p1516.

2. National Research Council. (1983) Little known Asian animals with a Promising Economic Future. Washington, D.C: National Academy Press. p46-48.

3. IUCN Red List of Threatened Species. http://www. iucnredlist.org/Accessed on 20-11-2013.

4. Mallon, D. P. (2008) "Antilope cervicapra". IUCN Red List of Threatened Species. Version 2012.2. International Union for Conservation of Nature.

5. Nowak, R. M. (1999). Blackbuck. Walker's Mammals of the World. The Johns Hopkins University Press, Baltimore, USA and London, UK. 1:1193-1194.

6. Sankar, K. Pabla, H. S., Patil C. K., Nigam P., Qureshi, Q., Navaneethan B., Manjrekar,M., Virkar, P. S., and Mondal, K (2013) Home range, habitat use and food habits of reintroduced gaur (Bos gaurus gaurus) in Bandhavgarh Tiger Reserve, Central India. Trop. Conserv. Sci., 6(1):50-69.

7. Nikunj, G and Nishith, D. (2011) Status, Occurrence, Distribution of Some Mammals of North Gujarath, India. Proce Zool Soc. 64 (1): 46-53.

8. Dellman, H.D. and Brown, E.M. (1981). Text Book of Veterinary Histology. Lea and Febieger, Philodolphia., p382-388.

9. Nagaraju, G. N, Prasad, R.V, Jamuna,K. V and Ramkrishna, V. (2012) Histological features in the differentiation of skin of spotted deer (Axis axis), cattle (Bos indicus) and goat (Capra hircus). Indian J. Vet. Anat., 24 (1): 10-12.

10. Shambhulingappa, Y. B., Prasad, R. V., Jamuna,K. V and Ramkrishna, V. (2013) Histomorphology of hair follicle pattern in wild big cats and sloth bear skin: a tool for forensic identification. J. Cell Tissue Res. 13(2):3679-3682.

11. Culling, C.F.A. (1981) Hand book of Histopathological and Histochemical Techniques. $3^{\text {rd }}$ edn, Butterworths, London and Boston. p85-86.

12. Singh, U. B. and Sulochana, S. (1996) Handbook of Histological and Histochemical techniques. $2^{\text {nd }}$ edn, Premier Publishing House, Hyderabad. p10-11.

13. Luna, L.G. (1968) Manual of Histological Staining Methods of the Armed Forces Institute of Pathology. $3^{r d} e d n$, Blakiston Div., McGraw-Hill book Co. New York. p. 25-78.

14. Raheem, M. H. A. and Al-Hety, M.S. (1997) Histological and morphometrical study of the skin of black goat. Iraqi J. Vet. Sci., 10(2): 59-71.

15. Atlee, B.A., Stannard, A.A., Fowler, M.E., Willemse, T., Ihrke, P.J. and Olivry, T. (1997) The histology of normal llama skin. Vet. Dermatol., 8 (3): 165-176.

16. Jenkinson, D.M and Nay, T. (1972) The sweat glands and hair follicles of Europ cattle. Aust. J. Boil. Sci., 25: 585-595.

17. Koul, G.L., Somvanshi, R. and Biswas, J.C. (1990) Follicle characteristics of non-wooly Indian goats. Res. Vet. Sci., 48:257-259.

18. Adib Moradi, M. and Sheibani, M.T. (2000) Histological study of hair follicles of Raini goat skin. J. Fac. Vet. Med., 55(2): 75-78.

19. Kapadnis, P.J., Bhosle, N.S. and Mugale, R.R. (2004) Hair follicle characters in Osmanabadi goats. Indian. J. Anim. Sci., 74(6): 623-625.

20. Hole, M. B., Bhosle, N.S. and Kapadnis, P.J. (2008) Study of hair follicles in Red Kandhari cows. Indian J. Anim. Res., 42(2): 151-152.

21. Lucy, K. M., Leena, C., Fathima, R. and Anneth, A. J. (2014) Anatomy of the skin of hippopotamus (Hippopotamus amphibious). 28 th Annual Convention of Indian Association of Veterinary Anatomists \& National Symposium, Bikaner, Rajasthan.p134.

$* * * * * * * *$ 